\title{
Getting astrophysical information from LISA data
}

\author{
R T Stebbinst, P L Bender† and W M Folknerł \\ $\dagger$ JILA, CB\#440, University of Colorado. Boulder, CO 80309, USA \\ ‡ Jet Propulsion Laboratory, California Institute of Technology, MS238-700 4800 Oak Grove \\ Drive, Pasadena, CA 91109 , USA
}

Received 19 November 1996, in final form 17 March 1997

\begin{abstract}
Gravitational wave signals from a large number of astrophysical sources will be present in the LISA data. Information about as many sources as possible must be estimated from time series of strain measurements. Several types of signals are expected to be present: simple periodic signals from relatively stable binary systems, chirped signals from coalescing binary systems, complex waveforms from highly relativistic binary systems, stochastic backgrounds from galactic and extragalactic binary systems and possibly stochastic backgrounds from the early Universe. The orbital motion of the LISA antenna will modulate the phase and amplitude of all these signals, except the isotropic backgrounds and thereby give information on the directions of sources.

Here we describe a candidate process for disentangling the gravitational wave signals and estimating the relevant astrophysical parameters from one year of LISA data. Nearly all of the sources will be identified by searching with templates based on source parameters and directions
\end{abstract}

PACS numbers: 0480N, 0705K, 9530S, 9555Y, 9575P, 9585S

\section{Introduction}

The sensitivity and operating band $(0.1 \mathrm{mHz}$ to $1 \mathrm{~Hz})$ of LISA are such that many types, and a very large number, of astrophysical sources should be detectable. The signals will vary greatly in character and strength. These many astrophysical signals make the scientific goals of the mission particularly attractive. However, their combination in the data stream presents a challenge for the data analyst. The goal of the analysis is to produce catalogues of gravitational wave sources with accurate estimates of their astrophysical parameters.

In this paper, we first discuss the signal types and their characteristics. Then, we present one possible scenario for the data flow and routine handling, strong-signal processing, weaksignal processing and background analysis. First, the strongest signals are identified in the spectrum, fitted in the time series and removed from the time series. Next, weaker periodic and chirped signals and poorly characterized signals (e.g. generic bursts) are searched for and removed. Then, weak signals with complex waveforms are sought with templates. For these signals, methods such as genetic algorithms, simulated annealing or hierarchical searches can be used to efficiently search the large parameter space. Finally, the spectrum of the remaining power can be analysed for continuous backgrounds. Those from galactic and extragalactic binaries can be modelled. Any isotropic residual signal not having the frequency dependence expected for extragalactic binaries would be a candidate for a background signal from the early Universe. 
The purpose of this paper is to provide an example of a general approach that could be used in analysing the LISA data for astrophysical information. We will not discuss the additional analyses that will be needed to assess the fundamental physics tests which LISA is capable of. Some of the techniques that will be discussed here have been studied and applied to real and/or simulated data from ground-based gravitational wave interferometers $[1,2]$.

\section{The nature of the signals}

The signals from expected sources can be roughly categorized into four main types: first, most detectable binary systems will generate simple periodic signals; secondly, pairs of intermediate mass black holes near coalescence will give chirped signals; thirdly, compact objects, such as small $\left(3-20 M_{\odot}\right)$ black holes, spiralling in highly relativistic orbits around massive $\left(10^{5}-10^{7} M_{\odot}\right)$ black holes in galactic nuclei, will generate very complex waveforms. Finally, there will be a stochastic background from close white dwarf binaries, both galactic and extragalactic, and there may possibly be a stochastic background from processes in the early Universe. These signals will exhibit a wide range of amplitudes. Coalescing massive black hole signals could, if present, exceed the weakest extractable signals by three orders of magnitude.

We will discuss these types of sources below to illustrate the range of signal characteristics and strengths expected in the LISA data. Note that we categorize the signals by their inherent spectral properties, but the orbital motion of the LISA antenna will modulate the phase of signals from all sources, and, in conjunction with the antenna sensitivity pattern and the source polarization, will modulate the amplitude $[3,4]$.

Additionally, as Thorne has emphasized [5], there may very well be exotic sources of gravitational waves, such as naked singularities and boson and soliton stars, which may be among the most interesting gravitational wave sources. Because the signals from these are generally not well understood, we do not consider them further inere.

\section{Simple periodic signals}

The simplest and most common signals expected in the LISA data stream will have constant frequency (except for detector motion). Binary systems of compact objects are expected to produce the bulk of the gravitational wave signals identifiable by LISA. Candidate systems will include neutron star binaries, close white dwarf binaries, interacting white dwarf binaries, cataclysmic variables and small black hole binaries $\left(<20 M_{\odot}\right)$. Very many of these systems in the Milky Way will be detectable. The strongest sources will be nearby in the galactic plane, but most will be toward the galactic centre. Verbunt has described these in his paper [6]. The number of galactic close white dwarf binaries should be so large that they form a continuum [7] below about $1 \mathrm{mHz}$ in a one year long data string, that is, there will be a confusion level where there are multiple sources per frequency bin of comparable amplitude, preventing the estimation of source parameters. The stronger galactic sources may rise as much as two orders of magnitude above this confusion level. Extragalactic sources of this type will be far more numerous and they will set the binary confusion noise level above roughly $3 \mathrm{mHz}$.

\section{Chirped signals}

Chirped signals, those whose frequency increases with time, are produced by the decaying orbits of coalescing objects. Extensive calculations of the expected signals over most of the 
time before coalescence are available. Matzner has discussed the computation of the signal strength during coalescence, when it becomes much more complicated.

If they exist, coalescing binaries of intermediate mass black holes $\left(\sim 10^{2}-10^{4} M_{\odot}\right)$ would produce observable signals around $1 \mathrm{mHz}$ and above. In some cases, these signals could exceed the instrument noise and confusion noise levels by as much as several hundred times, even out to a redshift of $z=1$.

A second possible source of chirped signals is massive $\left(\sim 10^{4}-10^{7} M_{\odot}\right)$ black hole binaries in the last year before coalescence. These might be the product of galactic mergers, as discussed by Rees [8] and Vecchio [9]. If these signals occur frequently enough to be seen, they would have signal-to-noise ratios of up to several thousand for sources at $z=1$.

\section{Complex waveforms}

Compact objects, such as small (3-20 $M_{\odot}$ ) black holes, can become trapped in highly relativistic orbits about massive black holes in galactic nuclei [10-12]. These orbits decay over decades or longer through the emission of gravitational radiation. The complex waveforms will have multiple periodicities. These sources may well be detectable at cosmological distances, but will have only moderate signal-to-noise ratios. They are particularly interesting because the signals should provide statistical information about physical conditions surrounding the massive black holes and about the mass function of small black holes.

\section{Stochastic backgrounds}

As mentioned above, the very large number of galactic and extragalactic binaries will, at some level, give an apparently stochastic background. In the region below about $1 \mathrm{mHz}$ where galactic binaries dominate, the background arises because there are typically several sources contributing at similar levels to a single frequency bin. Since most of these sources are confined to the galactic plane, this background will be spatially anisotropic. Between roughly 1 and $3 \mathrm{mHz}$, the galactic sources rapidly become sparse with increasing frequency, revealing an extragalactic background at a lower level. Above about $3 \mathrm{mHz}$ where mainly extragalactic sources occur, there are extremely many contributors to a frequency bin and the background is nearly isotropic.

The gravitational wave background from the Big Bang is very uncertain and the scenarios under which LISA could detect it are not well understood. However, detection would be a very important scientific benefit from LISA. If the cosmological gravitational wave background were large enough at $\mathrm{mHz}$ frequencies to be detected by LISA, then one would like to combine the results with those at other frequencies, such as from ground-based detectors, to determine the spectral shape. This might suggest which non-thermal process generated the background. In contrast with the galactic binary confusion level, one would expect the cosmological background to be quite isotropic.

\section{Data flow and routine handling}

To show how LISA data handling might proceed, we give a plausible scenario for the conventional processing of LISA data. Some processing of the LISA data must be done on the spacecraft in order to compress the data to fit within the telemetry bandwidth available for scientific data [13]. The beat signals from the interferometers will be recorded as a time series of phase measurements. Most of the orbital contributions to the signal will be 
removed using onboard information. The phase measurements will be corrected for laser and clock noise prior to compression for telemetry.

The ground processing of LISA data begins when the telemetry is received by the ESOC Mission Control Centre (figure 1). There the data will be catalogued and the payload and spacecraft housekeeping data will be separated from the scientific data. The payload and scientific data and relevant spacecraft parameters will be decompressed and forwarded to the LISA Science Centre for the scientific processing.

\section{LISA Data Flow}

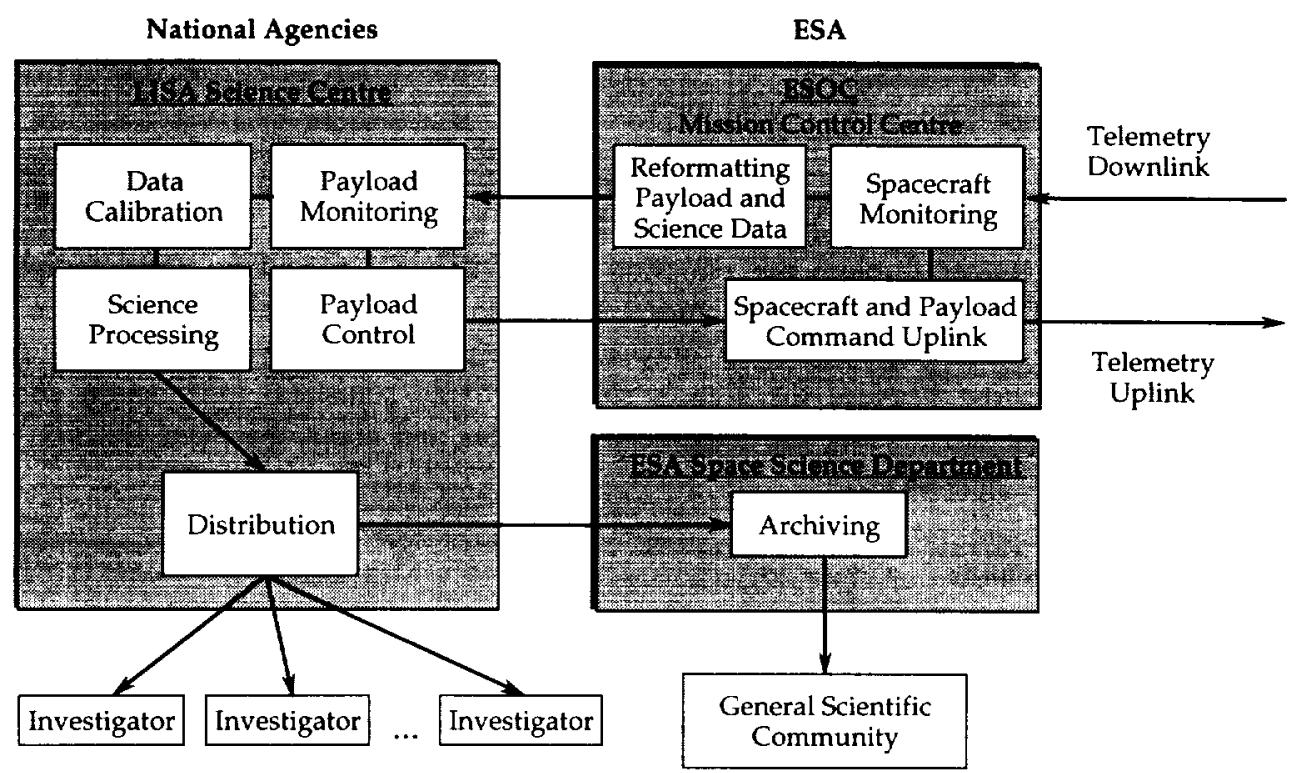

Figure 1. Routine data flow and handling. This flowchart illustrates how LISA data might flow from the receiving downlink site to the Science Centre and investigators.

At the Science Centre, the payload housekeeping parameters will be examined to track the health of the scientific payload. Quality assurance checks from housekeeping and external data will be used to determine limitations on the data. Preliminary quality assurance tests will also be performed on the scientific data to ensure that valid data are being received. Any necessary payload commands are sent back to the Operations Centre for uplinking to the spacecraft. The phase measurement time series will be converted to path length difference time series of several durations, say, week-, month- and year-long. Gaps will be filled where possible. Residual orbital effects will be removed from the data using ancillary information from the spacecraft and ground tracking. Phase jumps could possibly be recovered from the signals of the strongest sources. Strain time series of several durations and their Fourier transforms are calculated and stored as intermediate science products.

\section{Strong-signal processing}

There will be only a few gravitational wave sources which are known beforehand from electromagnetic observations. Comparisons of the actual signals with the predicted waveforms of known sources constitute a stringent test for the LISA instrument and the 
general theory of relativity. However, almost all of the gravitational wave sources will not have been observed previously. The general nature of their signals can often be anticipated, but the parameters of individual signals will have to be determined by searching over the space of possible parameters with templates.

For the purposes of this discussion, a strong signal is one whose parameters can be determined well enough by simple procedures to permit a preliminary subtraction of it from the data. For stronger signals, a good initial guess at the source parameters can be made by inspection of the power spectra. The direction parameters would be estimated at the same time as the source parameters.

The first step in separating the many periodic signals from astrophysical scurces in the LISA data will be identifying and removing the strong sources. The identification might proceed in one of two ways: the time series could be demodulated for each source direction resolution element and the spectra from the resulting time series examined for strong sources, or the astrophysical and source direction parameters for each strong source could be fitted in the original time string using the undemodulated spectrum to limit the parameter space of the templates. Although this choice deserves more study, we have tentatively chosen the latter approach for the present discussion, partly because the size of the effective direction resolution element depends on the strength of the source. In any event, the identification of strong periodic signals will produce estimates of the source direction, as well as the signal amplitude, frequency, eccentricity, polarization and phase. These signals will then be removed from the original time series.

The next step in the data processing is the identification and removal of the strong non-periodic signals, e.g. the chirped signals. For strong enough non-periodic sources, the parameter space can be significantly reduced by examining the original spectra and spectra based on subsets of the whole time series. There will also have to be a search for strong, unexpected types of sources with discovery filters.

The strong-signal processing phase will produce a catalogue of strong sources with estimates of their directions and the parameters of the emitting systems and an intermediate time series with the strong sources removed will be produced which will be the starting point for weak-signal processing.

\section{Weak-signal processing}

The search for weak signals will begin with an initial fitting of individual templates for periodic and chirped signals to the time series with the strong signals removed. This will involve a large number of pre-computed templates and will tentatively identify the weak sources. Then the fitted signals for the strong and the weak sources are combined and refit to the original time series. The results will then be catalogued and removed from the data set.

The second step will be to search for the complex signals, described above. The phase of these multi-periodic and quasi-periodic signals has information about the gravitational wave frequency, its time derivative, the radial orbital period, the azimuthal orbital period, the rate of precession of the node and the angular momentum of the massive black hole, in additional to the usual direction information. A brute force search would require a very large grid of templates and a more sophisticated search strategy is desirable.

Among the candidates for the search strategy are several based on a combination of 'steepest-descent' methods and random restarts in order to avoid local maxima in the parameter space. Two such approaches are the use of genetic algorithms [14] and simulated annealing [15]. Research on such approaches will certainly be needed. 
Another approach which is being investigated for ground-based gravitational wave searches is the hierarchical method. Since many of the parameters involve time, the number of templates increases very rapidly with the length of the data set. So, a hierarchical search would first fit templates computed over a coarser parameter grid to a shorter time series and then templates computed on a finer grid in the promising regions of parameter space would be applied to longer duration time series.

\section{Background analysis}

The binary confusion level is uncertain at this time because the space density of some types of contributors is poorly known $[6,7,16]$. Nonetheless, one can anticipate some of the properties of this continuum, and hence the approach to estimate it from the data. To begin with, the distribution of directions and signal strengths from resolved galactic binaries will be used to develop a statistical model for the distribution of different types of binaries throughout the galaxy. That model will then be extrapolated to somewhat lower and higher frequencies, with the help of whatever information is available on the initial period distribution and evolution of different types of close binaries. The predictions of the model for the galactic confusion level can be compared with the observations, after identified sources are removed, to possibly improve the model.

Next, an attempt would be made to fit the confusion noise due to extragalactic binaries, using knowledge of the frequency dependence of the spectral amplitude. If essentially all of the isotropic components can be fitted in this way, there will be no way to separate out a possible contribution from events in the early Universe, such as the Big Bang, phase transitions or vibrating cosmic strings. However, if the observed isotropic spectral amplitude does not fit that expected for extragalactic binaries, the difference would be a candidate for gravitational waves coming from the early Universe.

\section{Summary}

LISA is expected to search for a wide range of signal types with different characteristics. The data should be very rich in astrophysical information. We have outlined one possible approach to processing the data. The astrophysical parameters of strong and weak periodic and chirped signals will be estimated through the use of templates and removed from the data by least-squares fitting. Weak complex signals will then be searched for with templates. The parameter space, however, is very large. Thus the use of highly efficient search approaches, such as genetic algorithms, simulated annealing or hierarchical searches, must be investigated. The isotropic extragalactic background due to binaries and to possible events in the early Universe could be distinguished only by having different spectral signatures in the $\mathrm{mHz}$ frequency range. The source catalogues to be produced by LISA data will yield information about the space density throughout the galaxy of various types of compact binaries and hopefully also information about the distribution, evolutionary history and neighbourhoods of massive black holes.

\section{Acknowledgments}

Two of us (RTS and PLB) acknowledge support under NASA grant \#NAGW-4772. The research described in this paper was, in part, carried out by the Jet Propulsion Laboratory, 
California Institute of Technology, under a contract with the National Aeronautics and Space Administration.

\section{References}

[1] Schutz B 1991 The Detection of Gravitational Waves ed D Blair (Cambridge: Cambridge University Press) pp 406-52

[2] Schutz B 1996 Proc. 1995 Les Houches School Astrophys. Sources Gravitational Radiation ed J-A Marck and J-P Lasota (Berlin: Springer)

[3] Peterseim M, Jennrich O. Danzmann K and Schutz B F 1997 Class. Quantum Grav. 14 1507-12

[4] Jennrich O, Peterseim M. Danzmann K and Schutz B F 1997 Class. Quantum Grav. 14 1525-29

[5] Thome K 1995 Proc. Snowmass 95 Summer Study on Particle and Nuclear Astrophysics and Cosmology ed E Kolb and R Peccei (Singapore: World Scientific) pp 398-425

[6] Verbunt F 1997 Class. Quantum Grav. 14 1417-23

[7] Bender P and Hils D 1997 Class. Quantum Grav. 14 1439-44

[8] Rees M 1997 Class. Quantum Grav. 14 1411-15

[9] Vecchio A 1997 Class. Quantum Grav. 14 1431-7

[10] Hils D and Bender P 1995 Astrophys. J. $445 \mathrm{~L} 7$

[11] Sigurdsson S and Rees M 1997 Mon. Not. R. Astron. Soc. 284 318-26

[12] Sigurdsson S 1997 Class. Quantum Grav. 14 1425-9

[13] Stebbins R, Bender P, Folkner W and the LISA Science Team 1996 Class. Quantum Grav. 13 A285-9

[14] Davis L 1991 Handbook of Genetic Algorithms (New York: Van Nostrand)

Goldberg D 1989 Genetic Algorithms in Search, Optimization and Machine Learning (Reading, MA: AddisonWesley)

[15] Press W, Teukolsky S, Vetterling W and Flannery B 1992 Numerical Recipes 2nd edn, pp 436-48

[16] Hils D, Bender P and Webbink R 1990 Astrophys. J. 360 75-94 


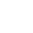

